\title{
Investigation of the Effect of Channel Structure and Flow Rate on On-chip Bacterial Lysis
}

\author{
Araz Norouz Dizaji ${ }^{1}$, Yasin Ozturk ${ }^{2}$, Hamed Ghorbanpoor ${ }^{1}$, Ahmet Cetak $^{3}$, Iremnur Akcakoca ${ }^{2}$, \\ Tanil Kocagoz ${ }^{4,5}$, Huseyin Avci ${ }^{6}$, Damion Corrigan ${ }^{7}$ and Fatma Dogan Guzel ${ }^{1 *}$
}

\begin{abstract}
Successful lysis of cells/microorganisms is a key step in the sample preparation in fields like molecular biology, bioengineering, and biomedical engineering. This study therefore aims to investigate the lysis of bacteria on-chip and its dependence on both microfluidic channel structure and flow rate. Effects of temperature on lysis on-chip were also investigated. To perform these investigations, three different microfluidic chips were designed and produced (straight, zigzag and circular configurations), while the length of the channels were kept constant. As an exemplary case, Mycobacterium smegmatis was chosen to represent the acid-fast bacteria. Bacterial suspensions of 1.5 McFarland were injected into the chips at various flow rates (0.6-8 $\mu \mathrm{l} / \mathrm{min})$ either at room temperature or $50^{\circ} \mathrm{C}$. In order to understand the on-chip lysis performance fully, off-chip experiments were carried out at durations which are equal to those bacteria spent in the channel from inlet to the outlet at different flow rates. We also performed COMSOL multiphysics program simulations to evaluate further the effect of the applied parameters. As a result, we found that the structure and the flow rate do not affect lysis over all in all investigated channel types, however on-chip experiments at room temperature produced more effective lysis compared to the on-chip and the off-chip samples performed at higher temperatures. Interestingly on-chip experiments at higher tempratures do not result in effective lysis.
\end{abstract}

Index Terms - Microfluidic chip, Thermal lysis, On-chip bacterial lysis, Mycobacterium smegmatis

\section{INTRODUCTION}

B acterial infections have continued to be a major cause of human morbidity and mortality [1]. Among them, tuberculosis and leprosy are categorized as acid-fast bacteria diseases since they could resist decolorization in acid, and these bacteria are responsible for millions of deaths each year and a great effort has been made by health institutions to control these

This study was conducted in the frame of a Newton Katip Celebi Fund between Turkey and UK and supported by Turkish Scientific and Technological Council under the grant number of 217S793.

A. Norouz Dizaji, H. Ghorbanpoor and F. Dogan Guzel are with the Department of Biomedical Engineering, Ankara Yildirim Beyazit University, Ankara, Turkey (e.mail: araz.norouz@gmail.com, hamedeghorbanpoor@gmail.com and fdogan@ybu.edu.tr).

Y. Ozturk and I. Akcakoca are with the Department of Material Engineering, Yildirim Beyazit University, Ankara, Turkey.

A. Cetak is with the Department of Mechanical Engineering, Yildirim Beyazit University, Ankara, Turkey. infections [2, 3]. Accurate and rapid detection of these infections is extremely important for the early treatment and control of these diseases [4]. There are currently many traditional approaches used as diagnostic methods, however most of these methods possess disadvantages in terms of time, cost and lower sensitivity $[4,5]$. In parallel with the advances in technology, new methods have emerged for rapid, cheap and effective diagnostics. Microfluidic-based lab-on-chip systems are among these methods [6-11]. These systems show many great advantages such as short assay time, cost reduction by miniaturization, integration, automation and parallelization of biochemical processes while enabling to work in microliter and even nanoliter volumes of substances [12]. With these systems, the genome level detections could also be done including the identification of possible mutations $[8,13]$.

Cell lysis (or cellular disruption) is the first and most important step in the analysis of bacteria at the molecular levels [14]. Cell lysis refers to the disintegration of cell wall in order to release the cytoplasmic compounds such as DNA, RNA and proteins prior to the analysis of interest [14]. Many conventional methods are commonly used for bacterial lysis such as mechanical, physical, chemical, enzymatic, thermal or combination of these techniques [14-18]. Thermal lysis is known as one of the most preferred techniques (in the physical lysis category) due to the obvious advantages like low cost, high throughput, easy application features and the simplicity of integration into microfluidic systems while eliminating the need for chemical agents $[14,18]$. There are limited relevant reports for the thermal lysis on-chip in the literature [18, 19]. On-chip thermal lysis has been more commonly employed due to the fact that it decreases the assay steps and eventually assay time, cost, and sample consumption in addition to provide portability for on-field applications.

In this study, we aimed to investigate the efficiency of thermal bacterial lysis on-chip using different microfluidic channel

T. Kocagoz is with the Department of Medical Biotechnology, Institute of Health Sciences and Department of Medical Microbiology, School of Medicine, Acibadem Mehmet Ali Aydinlar University, Istanbul, Turkiye.

H. Avci is with the Metallurgical and Materials Engineering Department, Eskisehir Osmangazi University, Eskisehir, Turkey.

Damion Corrigan is with the Department of Biomedical Engineering, University of Strathclyde, 40 George Street, Glasgow G1 1QE, United Kingdom.

* Corresponding author: F. Dogan Guzel, e-mail: fdogan@ybu.edu.tr 
structures and flow rates both at room temperature and $50{ }^{\circ} \mathrm{C}$. The schematic illustration of the on-chip lysis in our set-up is given in Fig. 1. As a case study to acid-fast bacterial infections (tuberculosis and leprosy), M. smegmatis was chosen since it is a non-pathogenic strains of Mycobacteria spp. so it is safe to work with to minimize the contagious risks in a classical lab environment. Even though M. smegmatis is one of the nonpathogenic strains, it has structurally and genetically high similarities with other pathogenic Mycobacteria which makes it a suitable cell model [20,21].

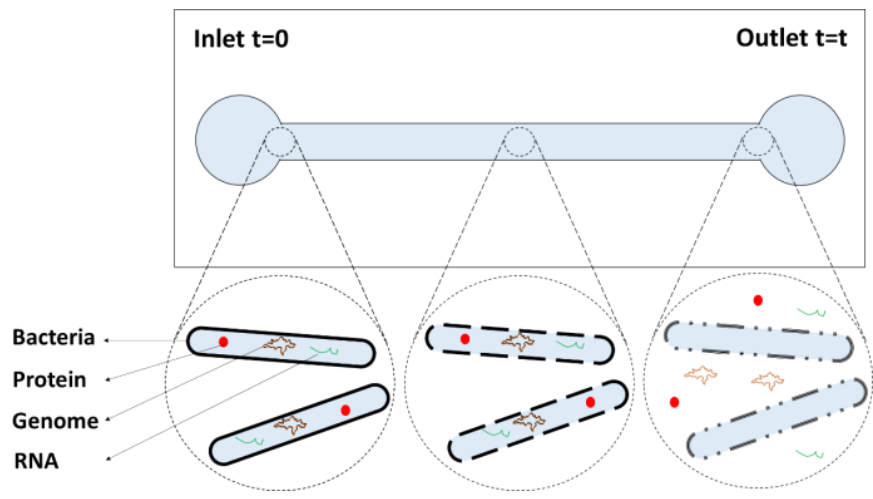

FIG. 1. Schematic illustration of thermal on-chip bacterial lysis throughout the channel.

Polydimethylsiloxane (PDMS)-based microfluidic structures were designed and fabricated in a clean room environment using classical soft lithography techniques. Different flow rates were examined and compared at the same time durations in offchip lysis experiments. The on-chip results were compared to the off-chip findings and further evaluation was made with COMSOL multiphysics program simulations. It was found that lysis performance on-chip showed approximately 10-fold increase at room temperature whereas on-chip thermal lysis results were similar to that off-chip samples. It is well known that pressure is proportional to the microchannel length therefore increase in channel length could cause increase in pressure that directly affect bacterial lysis. However, increase in temperature could drop viscosity and it should be noted that this causes decrease in pressure as well. We believe that our versatile and robust system of microfluidic on-chip lysis of cells and/or bacteria will widely used in various applications of PCR, biosensors, antibiotic susceptibility testing, etc. with different temperatures $\left(>50{ }^{\circ} \mathrm{C}\right)$ and exposure times (seconds to hours). These parameters can easily be modified by using a microfluidic set-up to investigate different conditions.

\section{MATERIALS AND METHODS}

\section{A. Production of the microfluidic chip}

Three microfluidic chip whit different structures were designed in size of $25 \times 75 \mathrm{~mm}$ (straight, zigzag, and circular). The length and depth of designed microchannels were $1.20 \mathrm{~m}$ and $50 \mu \mathrm{m}$, respectively. The total volume of each chip was designed to be about $6 \mu 1$.

AutoCAD program were used to design michrochannels, and the photomasks were produced by Cozum-Tanitim, Turkey.
Production of chips were carried out using a conventional soft lithography technique [22]. Briefly, in order to casting of PDMS (Sylgard 184, Dow Corning, USA) on silicon wafer, negative SU-8 2050 photoresist (Nippon Kayaku, MicroChem, Japan) mold was prepared at a ratio of 10:1. It was then baked for 6 hours at $60{ }^{\circ} \mathrm{C}$ followed by peeling off the patterned PDMS. PDMS chips were then cut using a razor blade. $1 \mathrm{~mm}$ wide inlets and outlets were opened using a punch-opener (Selles Medical, UK) and a scotch tape was used to remove any remaining particles on the PDMS. Glass slides and were sonicated in Acetone (Sigma-Aldrich, USA), then in Ethanol (Sigma-Aldrich, USA) for short time, and then washed with $\mathrm{dH}_{2} \mathrm{O}$ and dried with $\mathrm{N}_{2}$ gas. Both PDMS chips and glass slides were then treated with Air Plasma and subsequently bonded together, and left on a hot plate to improve the bonding.

\section{B. Bacterial culture}

M. smegmatis strain (ATCC 14468) was provided by Acibadem University Hospitals (Turkey) and was cultured in 5 $\mathrm{ml}$ of Middlebrook 7H9 medium (Sigma-Aldrich, USA) that was supplemented with $0.5 \%$ of Tween (Sigma-Aldrich, USA) and $2 \%$ of glycerol (Sigma-Aldrich, USA) to eliminate coagulation. Cultured bacteria were incubated in sterile glass tubes containing $5 \mathrm{ml}$ medium at $37^{\circ} \mathrm{C}$ until they reached the exponential growth phase. Propagated bacteria were transferred to sterile microtubes, centrifuged (Daihan, South Korea) for 5 min at $4000 \mathrm{rpm}$. The supernatant was removed and an equal amount of the fresh medium was added on precipitated bacteria.

\section{Off-chip experiments}

In this study, off-chip experiments were carried out at different temperatures and durations at a fixed temperature. At first, M. smegmatis bacteria were separately kept at $25,50,60$, $70,80,90$ and $96{ }^{\circ} \mathrm{C}$ for $20 \mathrm{~min}$ to find the optimal temperature range. Secondly, bacteria were kept at $50{ }^{\circ} \mathrm{C}$ for a particular period of time. For this purpose, the M. smegmatis bacteria at maximum concentration were transferred to a sterile screw-cap $1 \mathrm{~mL}$ glass vials. The vials were then kept at $50^{\circ} \mathrm{C}$ for a particular time period. Afterward, the solutions were transferred to microtubes and centrifuged at $10000 \mathrm{rpm}$ for $10 \mathrm{~min}$. Supernatants were analyzed using NanoDropTM 2000/2000c spectrophotometer (Thermo Scientific ${ }^{\mathrm{TM}}$, USA) for the measurement of DNA concentrations.

\section{On-chip experiments}

Polyethylene tubing with $1 \mathrm{~mm}$ outer diameter (Harvard Apparatus, USA) were inserted into the inlets and outlets of the integrated PDMS chips. $M$. smegmatis bacteria that adjusted to 1.5 McFarland, using a densitometer device (Biosan, Lativa), were taken into a $1 \mathrm{ml}$ volume syringe and injected into the channel inlets through the tubings at different flow rates. Lysed bacteria were collected from the outlet of the channels into a vial. The thermal lysis experiments were carried out on a heater (Daihan, South Korea) at $50{ }^{\circ} \mathrm{C}$ in the same manner. All experiments were repeated three times. The collected bacterial solutions were centrifuged at $10000 \mathrm{rpm}$ for 10 minutes and supernatants were analyzed using the NanoDrop ${ }^{\mathrm{TM}} 2000 / 2000 \mathrm{c}$ spectrophotometer to measure the DNA concentrations. 


\section{E. COMSOL simulation studies}

COMSOL Multiphysics program (COMSOL Multiphysics ${ }^{\circledR}$ Modeling Software 5.4, country) was used to simulate the experiments. The 2D CAD designs of the chips were transferred to the program. Simulation parameters were defined using the laminar flow module. Mesh properties were calibrated according to the fluid dynamics. Rectangular mesh type and triangular mesh were used on the walls and inside the channel, respectively. All simulations were carried out at a flow rate of $0.6 \mu \mathrm{l} / \mathrm{min}$ and at temperatures of $25^{\circ} \mathrm{C}$ and $50{ }^{\circ} \mathrm{C}$. Water was chosen as the fluidic medium.

\section{RESULTS AND DISCUSSION}

\section{A. Experimental studies}

The main aim of this study was to investigate the on-chip thermal lysis efficiency of microfluidic chips with different designs and applied varius flow rates. Therefore, the experiments were carried out on-chip at room temperature and $50{ }^{\circ} \mathrm{C}$, then compared with the off-chip results. Three different microfluidic chips were designed in straight, zigzag and circular shapes, as shown in Fig. 2(a), Fig. 2(b) and Fig. 2(c), respectively (Photos of the produced chips are given in Fig. S1).

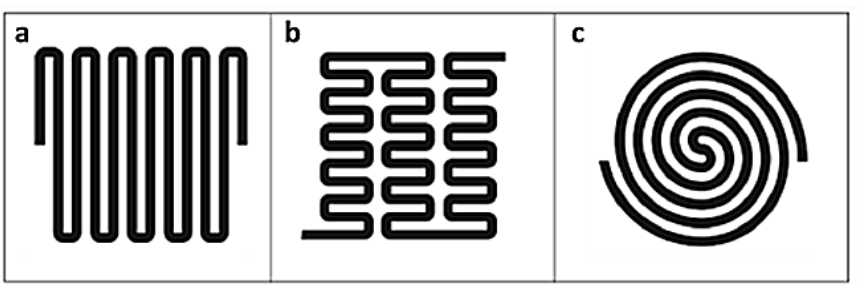

FIG. 2. Representative 2D images of the channel structures. (a) Straight, (b) Zigzag and (c) Circular.

The reasons for the choice of these designs was to compare and better understand the effect of the frequency with the degree of bend regions in the microfluidic channels on the movement and disintegration of the bacterial cell wall. We presumed that changes due to the pressure drop and velocity flow profiles at the bend regions would greatly affect the dynamics of bacteria while passing through the channel, as also mentioned in literature [23]. The determination of the degree of the effect would then facilitate further studies. The off-chip lysis durations were determined according to the confinement times of bacteria solution inside the microfluidic channels, as illustrated in Table 1.

TABLE I

FLOW RATES AND EQUIVALENT CONFINEMENT DURATIONS IN THE CHANNEL - OFF-CHIP LYSIS DURATIONS.

\begin{tabular}{cc}
\hline Flow rate $(\mu \mathrm{l} /$ min) & Duration $(\min )$ \\
\hline 0.6 & $32^{\prime}: 30^{\prime \prime}$ \\
1 & $20^{\prime}: 50^{\prime \prime}$ \\
2 & $10^{\prime}: 20^{\prime}$ \\
4 & $5^{\prime}: 25^{\prime \prime}$ \\
6 & $2^{\prime}: 10^{\prime \prime}$ \\
8 & $1^{\prime}: 05^{\prime \prime}$ \\
\hline
\end{tabular}

Though there are many methods such as re-culturing of lysate, gel-electrophoresis or commercial kits to count released genome content $[24,25]$. In this study, to evaluate the lysis efficiency, the genomic DNA concentration released into the lysate as a result of systematic variation of the lysis conditions was monitored as an indicator of efficiency using a NanoDrop device to minimize the time and cost.

The results of the off-chip experiments are shown in Fig. 3. Fig. 3(a) represents the off-chip lysis at different temperatures while Fig. 3(b) shows the off-chip thermal lysis at varying lysis durations at $50{ }^{\circ} \mathrm{C}$. Although lysis of Mycobacterium at a temperature above $80{ }^{\circ} \mathrm{C}$ is recommended by many groups, there are some reports in the literature that lysis were effectively performed at lower temperatures and durations [24, 26-28]. According to our results, about a 10-fold increase in DNA concentration was observed due to increasing of temperature to $50{ }^{\circ} \mathrm{C}$ (from room temperature). There was no significant increase in DNA concentration between $50{ }^{\circ} \mathrm{C}$ and $96{ }^{\circ} \mathrm{C}$. Based on the obtained results, it was determined that heating at $50^{\circ} \mathrm{C}$ was sufficient for effective lysis during on-chip experiments. In this way, any evaporation problem by using the on-chip technique was minimized as well, which is a known consequence of the permeability of PDMS. Fig. 3(a) shows higher lysis efficiency compared to Fig. 3(b). This is due to the differences in the starting bacteria concentration that chosen for the experimentation. Albeit the difference, as demonstrated by the trends in the graphs can still be used as an evaluator for the on-chip systems, therefore the experiments were not repeated at the same concentrations. As seen from Fig 3(b), off-chip experiments performed at $50{ }^{\circ} \mathrm{C}$ showed an increase in the nucleic acid content with an increase in the duration. This result is in line with the literature [14,29]. The highest lysis efficiency was obtained at about 32 minutes and produced a nucleic acid yield of approximately $2.5 \mathrm{ng} / \mu 1$.

In comparison, Fig. 4(a) and Fig. 4(b) shows the on-chip results obtained at room temperature and $50{ }^{\circ} \mathrm{C}$, respectively. The results indicate that there was not a significant change in onchip lysis performance both at room temperature and with heating, when the flow rates were varied in different chip designs. This clearly means that the lysis efficiency is independent of the design and flow rate within the given conditions. It is possible that the chosen channel length is long enough to lyse the bacteria effectively in any case and one may question whether shorter channel lengths would have an impact on the lysis efficiency. Considering the on-chip lysis durations (1 min to $30 \mathrm{~min}$ ), the chosen channel length and the flow rates seemed to be reasonable for the investigation. Another finding was that there was an almost 10-fold enhancement in the DNA content at room temperature, raising the DNA concentration to about $12 \mathrm{ng} / \mu \mathrm{l}$. This implies that the flow rate has an impact on the lysis process although the lysis is not directly affected by fluid velocity and channel structure. The amount of obtained DNA due to on-chip lysis in this study is close to the amount of DNA yield obtained by Jitae Kim et al., using the CD microfluidic lysis method obtained from bacterial concentration of about $\mathrm{OD}_{600}=2.0$ [30]. M. Dominika Kulinski et al. 
performed bacterial lysis with the silica impregnated polymer monolithic columns and the obtained DNA concentration was about $340-480 \mathrm{ng} / \mathrm{ml}$ as a result of the lysis $10^{1}-10^{5} \mathrm{cfu} / \mathrm{ml}$ bacteria [31]. In another study, Md. Shehadul Islam et al., performed electrical lysis of bacteria in a microfluidic device using a nanoporous membrane. According to their results, the DNA concentration obtained after lysis was achieved below 10 $\mathrm{pg} / \mu \mathrm{l}$ for a bacterial concentration of about $10^{8}-10^{9} \mathrm{cfu} / \mathrm{ml}[32]$. Though types of bacteria is different, compared to these studies, the amount of DNA obtained by our method seemed to be comparable and/or higher.
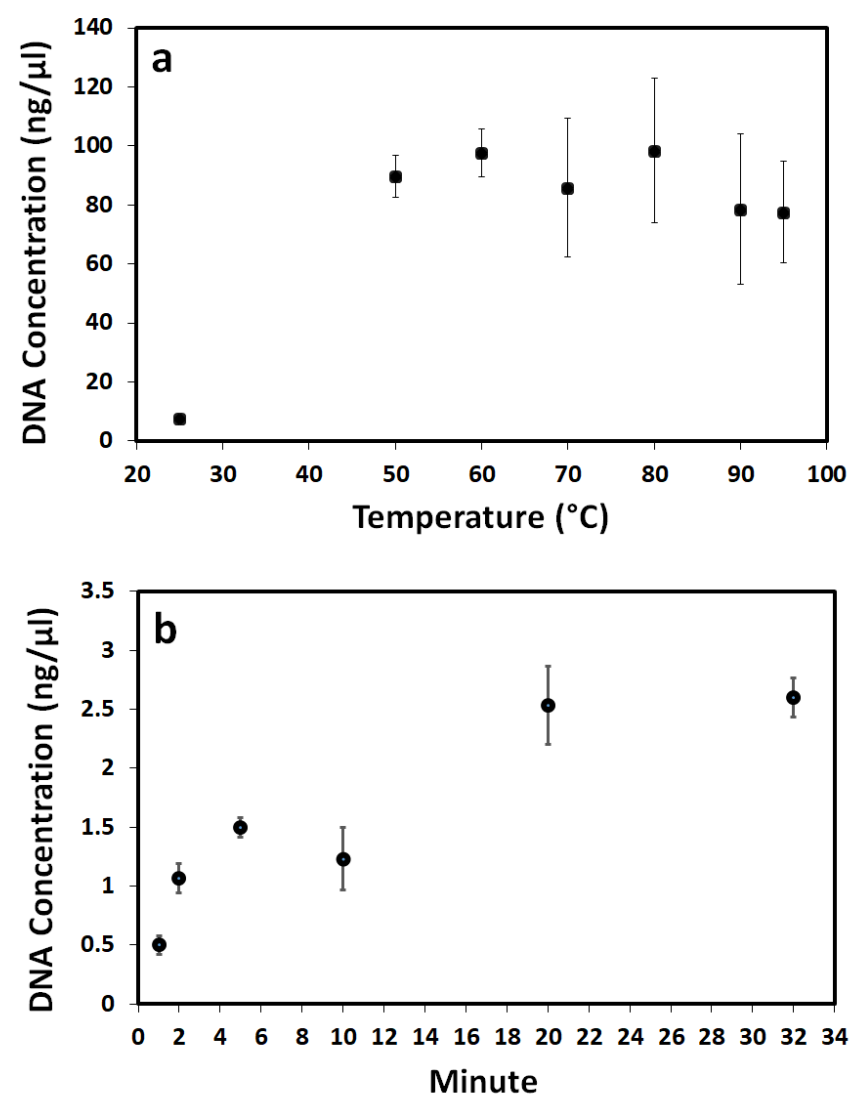

FIG. 3. Off-chip lysis performance of $M$. smegmatis at different temperatures and durations (Bacterial concentration used at maximum level). (a) Off-chip lysis performance of M. smegmatis at different temperatures. (b) Off-chip lysis performance of $M$. smegmatis at different durations at $50{ }^{\circ} \mathrm{C}$.

Interestingly it was also found that the on-chip lysis experiments showed a decrease in lysis performance at $50{ }^{\circ} \mathrm{C}$, as seen in Fig. 4(b). We presume that one of the main reasons is the physical and mechanical properties of PDMS change with temperature and the PDMS becomes softened and stretched at the increased temperatures [33]. Another reason is probably due to the fact that the increase in temperature decreases the viscosity of the fluid inside, which then consequently causes the pressure to decrease [34]. Another observation was that on-chip thermal lysis performance dropped with increases in the flow rates at $50^{\circ} \mathrm{C}$. This is possibly due to the fact that bacteria were exposed to heating for a shorter period of time with the increase in the flow rates. Therefore, the effect of heating on on-chip lysis was reduced as expected [18]. However, it is striking to
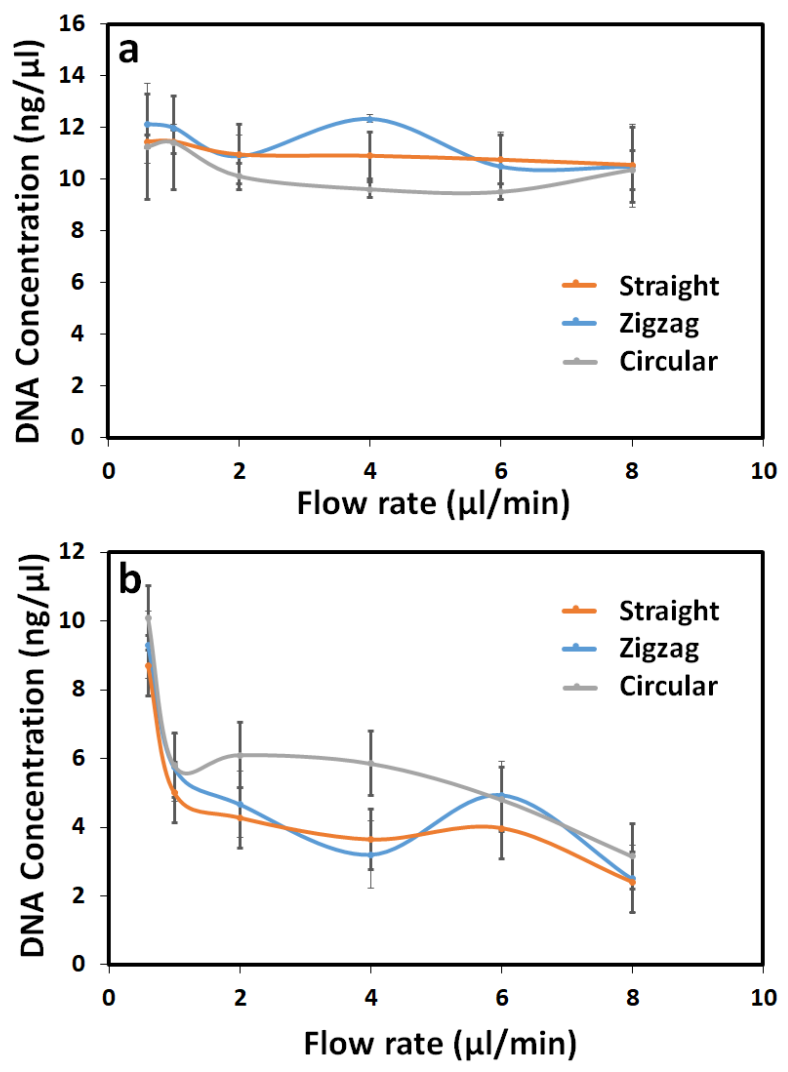

FIG. 4. Off-chip lysis performance of $M$. smegmatis using different flow rates and channel designs at different temperatures. (a) On-chip lysis at room temperature and (b) at $50{ }^{\circ} \mathrm{C}$ at varying flow rates.

see that the lysis efficiency becomes similar to the off-chip results even with the reduction.

When the on-chip and off-chip statistics were compared in terms of lysis duration, it can be seen that lysis can occur effectively within even a duration of $1 \mathrm{~min}$. Therefore we can conclude that our on-chip experiments are more efficient for the lysis process.

\section{B. Simulation studies}

In order to provide a better interpretation of the on-chip lysis results, the experiments were simulated for the different designs and temperatures (room temperature and $50{ }^{\circ} \mathrm{C}$ ). The representative pressure distributions for a caption of the whole designs were shown in Fig. 5. It was seen that the main difference in the pressure distribution among the designs occurs at the twisting regions along the microchannels and this results in sudden pressure changes. The peak pressure is estimated to be about $2.94 \mathrm{kPa}$ for a flow rate of $0.6 \mu \mathrm{l} / \mathrm{min}$ at room

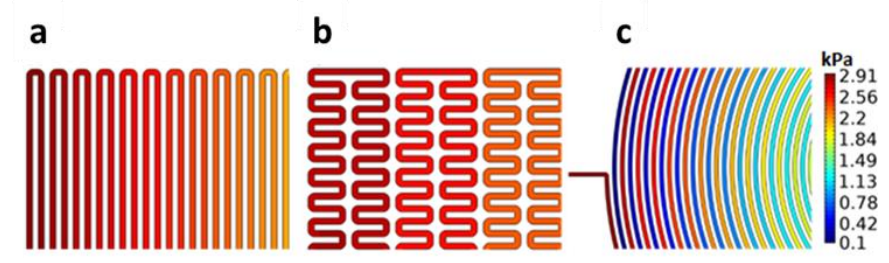

temperature and it occurs mostly at the inlet of the channels.

FIG 5. Illustration of pressure distribution for each design at room temperature; (a) Straight channel design, (b) Zigzag channel design, (c) Circular channel design. 
We assume that the overall effect of the pressure distribution on lysis efficiency stays almost the same for all designs although partial distributions behave differently. As also seen from simulations, the pressure drop is high at the beginning of the fluid intake while it drops towards the outlet as indicated in the literature [35].

Figure 5 shows the graph of the pressure drops for each design with a similar starting point. In straight and zigzag designs, the pressure drops across the microchannels were recorded similarly while at the 90 degree twists the distribution varies. This is shown in Fig. 6(a) and Fig. 6(b), respectively. There was a steady change in twisting regions unlike 90 -degree bends in circular design compared to the others, as shown in Fig. 6(c). As seen in Fig. 6, high inlet pressure at room temperature also means that the pressure drops progressing along the way is high whereas it is possible to observe the lower pressure drop with heating.

Simulations also lead to an understanding of the effect of changing viscosity with heating. Due to the decrease in fluid viscosity as a result of increased temperature, the inlet pressure decreased in the microchannels.

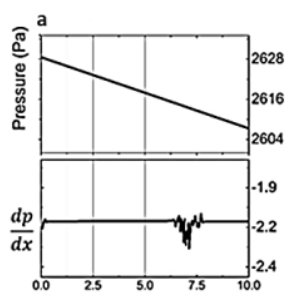

Arc Length (mm)

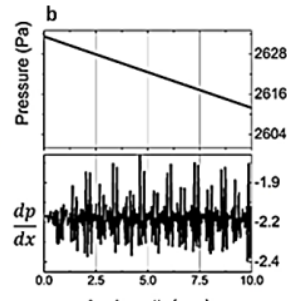

Arc Length (mm)
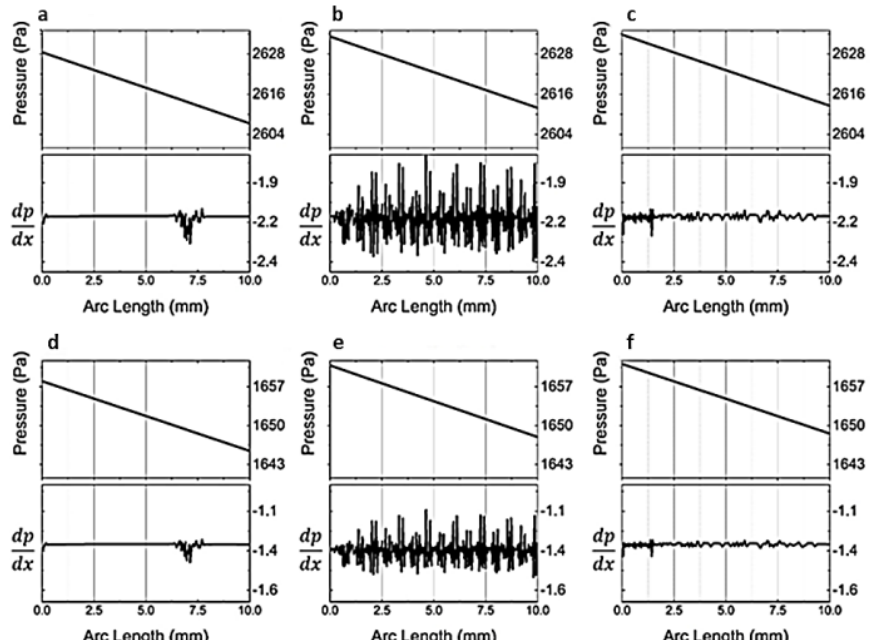

Arc Length (mm)

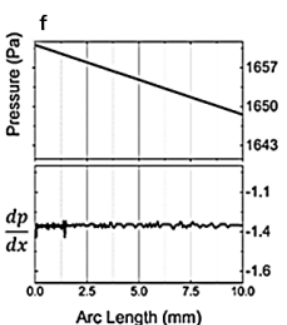

Arc Length $(\mathrm{mm})$
FIG. 6. The pressure drop along the microchannels and the derivative of pressure along the channels $(\mathrm{dp} / \mathrm{dx})$ for observing characteristics of bend regions along the channels. (a), (b) and (c) corresponds to $25{ }^{\circ} \mathrm{C}$ medium temperature of straight, circular and zigzag design, respectively. (d), (e) and (f) corresponds to $50{ }^{\circ} \mathrm{C}$ medium temperature of the same designs, respectively.

According to the simulations, we observed that the inlet pressure decreases by $56 \%$ when the temperature rises from 25 ${ }^{\circ} \mathrm{C}$ to $50{ }^{\circ} \mathrm{C}$ for all designs. This analysis supports the decrease in bacterial lysis performance due to temperature increase during the on-chip experiment. In studies reported in the literature, it was observed that relatively high pressures (25-30 $\mathrm{kPa})$ need to cell deformation and lysis in microchannels [36]. However, based on our results, even lower pressures $(3 \mathrm{kPa})$ are sufficient for an efficient lysis process. The viscosity and temperature relationship exerted on water was tabulated by Kestin and Wakeham [37]. It is now the common known fact that there is an inverse relationship between viscosity and temperature [38]. This means the viscosity of water was strictly decreased with increasing temperature. From another angle, viscosity can also be linked to the pressure drop with the increasing pressure, the viscosity of the fluid increases, though the pressure drop is affected by other parameters. A good approximation for the analytical modelling of pressure drop exerted on rectangular shaped-channel microfluidics was constructed by Bahrami et al. [39].

$$
d p=\frac{\mu \varepsilon L Q}{4 c^{4}\left(1 / 3-64 \varepsilon t / \pi^{5}\right)}
$$

In this formula, $d p$ represents pressure drop, $\mu$; viscosity, $\varepsilon$ : aspect ratio (height/width), L; length of microchannels, t: tanh $(\pi / 2 \varepsilon)$ and $\mathrm{c}$ represent half-height of the channel. In the light of this formulation, pressure and the viscosity characteristics can be modeled along a straight channel (L) with a stable flow rate $(\mathrm{Q})$ under the conditions of fully developed laminar flow [40]. It is also the fact that the bending curves (i.e 90 degree) can be added to the total amount of pressure drop $\left(\Delta p_{t o t}\right)$ and results in local pressure change along the microchannels [41]. The velocity profile of the fluid flow at the bending regions would also be different and contributing to the results. However, in general, our experimental evidence suggests that the overall effect of the changes in pressure drops, thus velocity profiles and viscosity, on lysis performance eventually stays the same. The only observable difference that can be directly linked to the simulations is the decrease in lysis efficiency when the heating is applied.

\section{CONCLUSION}

In this study, three different microfluidic chips were designed and fabricated for the investigation of on-chip bacterial lysis performance. The conventional photolithographic technique was applied to fabricate microfluidic chips for the desired structures. For off-chip experiments, M. smegmatis was cultured and heated at various temperatures between $25-96{ }^{\circ} \mathrm{C}$ for $20 \mathrm{~min}$, separately. The result shows that heating at $50{ }^{\circ} \mathrm{C}$ was sufficient for bacterial lysis. As for the on-chip experiments, the bacteria were injected parallelly to the prepared chips with different flow rates $(0.9,1,2,4,6$ and 8 $\left.\mu \mathrm{m} . \mathrm{min}^{-1}\right)$. These experiments were carried out in three replications both at room temperature and at $50{ }^{\circ} \mathrm{C}$. Off-chip experiments were carried out within a time period equal to the on-chip durations at different flow rates. Even in the room temperature, on-chip results showed significant bacterial lysis efficiency compare to off-chip results given at $50^{\circ} \mathrm{C}$. Moreover, the lysis on-chip without heating occurs within a much shorter duration than the off-chip experiments. This was due to the high pressure inside the channels. During the on-chip experiments, significant decreases were observed in the amount of lysis occurred with the temperature increase. COMSOL studies also supported the obtained results. The role of several parameters such as viscosity and type of bacteria can be investigated further under different conditions. It is believed that we present a highly efficient and universal lysis strategy at even room temperature occurring within a $1 \mathrm{~min}$ and the presented results here would facilitate the future on-chip lysis studies, setting a fundamental foundation for the development of integrated hybrid diagnostic devices.

\section{DISCLOSURE STATEMENT}

No potential conflict of interest was reported by the authors. 


\section{SUPPLEMENTARY MATERIAL}

The supplementary material file contains Fig. S1 showing the Photography of fabricated microfluidic chips due to the photolithography technique.

\section{ACKNOWLEDGMENT}

This study was conducted in the frame of a Newton Katip Celebi Fund between Turkey and UK and supported by the Turkish Scientific and Technological Council under the grant number of 217S793. Authors would like to thank Central Laboratory of Ankara Yildirim Beyazit University for allowing us to use NanoDrop in particular and other facilities, and Dr. I. Agah Ince for useful discussions.

\section{REFERENCES AND FOOTNOTES}

\section{REFERENCES}

[1] W. W. Stead, K. D. Eisenach, M. D. Cave, M. L. Beggs, G. L. Templeton, C. O. Thoen, and J. H. Bates, "When did Mycobacterium tuberculosis infection first occur in the New World? An important question with public health implications," American journal of respiratory and critical care medicine, vol. 151, no. 4, pp. 1267-1268, 1995.

[2] N. R. Meier, M. Jacobsen, T. H. Ottenhoff, and N. Ritz, "A systematic review on novel mycobacterium tuberculosis antigens and their discriminatory potential for the diagnosis of latent and active tuberculosis," Frontiers in immunology, vol. 9, pp. 2476, 2018.

[3] H. Bloch, "Acid-fast bacteria," Annual Reviews in Microbiology, vol. 7, no. 1, pp. 19-46, 1953.

[4] F. A. Al-Zamel, "Detection and diagnosis of Mycobacterium tuberculosis," Expert review of anti-infective therapy, vol. 7, no. 9, pp. 1099-1108, 2009

[5] J. J. Dunn, J. R. Starke, and P. A. Revell, "Laboratory diagnosis of Mycobacterium tuberculosis infection and disease in children," Journal of clinical microbiology, vol. 54, no. 6, pp. 1434-1441, 2016.

[6] J. Mairhofer, K. Roppert, and P. Ertl, "Microfluidic systems for pathogen sensing: a review," Sensors, vol. 9, no. 6, pp. 4804-4823, 2009.

[7] K. F. Lei, "Microfluidic systems for diagnostic applications: A review," Journal of laboratory automation, vol. 17, no. 5, pp. 330-347, 2012.

[8] C. Lui, N. C. Cady, and C. A. Batt, "Nucleic acid-based detection of bacterial pathogens using integrated microfluidic platform systems," Sensors, vol. 9, no. 5, pp. 3713-3744, 2009.

[9] E. O. Blair, S. Hannah, V. Vezza, H. Avc1, T. Kocagoz, P. A. Hoskisson, F. D. Güzel, and D. K. Corrigan, "Biologically modified microelectrode sensors provide enhanced sensitivity for detection of nucleic acid sequences from Mycobacterium tuberculosis," Sensors Actuators Reports, pp. 100008, 2020.

[10] F. D. Guzel, and H. Avci, "Fabrication of nanopores in an ultra-thin polyimide membrane for biomolecule sensing," IEEE Sensors Journal, vol. 18 , no. 7, pp. 2641-2646, 2018.

[11] F. D. Güzel, and B. Miles, "Development of in-flow label-free single molecule sensors using planar solid-state nanopore integrated microfluidic devices," Micro Nano Letters, vol. 13, no. 9, pp. 1352$1357,2018$.

[12] S. Halldorsson, E. Lucumi, R. Gómez-Sjöberg, and R. M. Fleming, "Advantages and challenges of microfluidic cell culture in polydimethylsiloxane devices," Biosensors and Bioelectronics, vol. 63, pp. 218-231, 2015.

[13] B. Zribi, E. Roy, A. Pallandre, S. Chebil, M. Koubaa, N. Mejri, H. Magdinier Gomez, C. Sola, H. Korri-Youssoufi, and A.-M. HaghiriGosnet, "A microfluidic electrochemical biosensor based on multiwall carbon nanotube/ferrocene for genomic DNA detection of Mycobacterium tuberculosis in clinical isolates," Biomicrofluidics, vol. 10, no. 1, pp. 014115, 2016.
[14] M. Shehadul Islam, A. Aryasomayajula, and P. R. Selvaganapathy, "A review on macroscale and microscale cell lysis methods," Micromachines, vol. 8, no. 3, pp. 83, 2017.

[15] P. E. Vandeventer, K. M. Weigel, J. Salazar, B. Erwin, B. Irvine, R. Doebler, A. Nadim, G. A. Cangelosi, and A. Niemz, "Mechanical disruption of lysis-resistant bacterial cells by use of a miniature, lowpower, disposable device," Journal of clinical microbiology, vol. 49, no. 7, pp. 2533-2539, 2011.

[16] B. Pethica, "Lysis by physical and chemical methods," Microbiology, vol. 18 , no. 2, pp. 473-480, 1958.

[17] O. Salazar, and J. A. Asenjo, "Enzymatic lysis of microbial cells," Biotechnology letters, vol. 29, no. 7, pp. 985-994, 2007.

[18] M. M. Packard, E. K. Wheeler, E. C. Alocilja, and M. Shusteff, "Performance evaluation of fast microfluidic thermal lysis of bacteria for diagnostic sample preparation," Diagnostics, vol. 3, no. 1, pp. 105116, 2013.

[19] M. Mahalanabis, H. Al-Muayad, M. D. Kulinski, D. Altman, and C. M. Klapperich, "Cell lysis and DNA extraction of gram-positive and gram-negative bacteria from whole blood in a disposable microfluidic chip," Lab on a Chip, vol. 9, no. 19, pp. 2811-2817, 2009.

[20] J. S. Tyagi, and D. Sharma, "Mycobacterium smegmatis and tuberculosis," Trends in microbiology, vol. 2, no. 10, pp. 68-69, 2002.

[21] J.-M. Reyrat, and D. Kahn, "Mycobacterium smegmatis: an absurd model for tuberculosis?," Trends in microbiology, vol. 9, no. 10, pp. 472-474, 2001.

[22] F. D. Guzel, and F. Citak, "Development of an on-chip antibiotic permeability assay with single molecule detection capability," IEEE transactions on nanobioscience, vol. 17, no. 2, pp. 155-160, 2018.

[23] J. You, L. Flores, M. Packirisamy, and I. Stiharu, "Modeling the effect of channel bends on microfluidic flow," IASME Trans, vol. 1, no. 1, pp. 144-151, 2005.

[24] P. Shetty, D. Ghosh, and D. Paul, "Thermal lysis and isothermal amplification of Mycobacterium tuberculosis H37Rv in one tube," Journal of microbiological methods, vol. 143, pp. 1-5, 2017.

[25] C. Gill, J. H. van de Wijgert, F. Blow, and A. C. Darby, "Evaluation of lysis methods for the extraction of bacterial DNA for analysis of the vaginal microbiota," PloS one, vol. 11, no. 9, 2016.

[26] W. J. Valente, E. Pienaar, A. Fast, A. Fluitt, S. E. Whitney, R. J. Fenton, R. G. Barletta, O. Chacon, and H. J. Viljoen, "A kinetic study of in vitro lysis of Mycobacterium smegmatis," Chemical engineering science, vol. 64, no. 9, pp. 1944-1952, 2009.

[27] M. Miyata, A. C. B. Santos, N. H. Mendes, E. A. Cunha, F. A. F. d. Melo, and C. Q. F. Leite, "Assessment of the quality of dna extracted by two techniques from Mycobacterium tuberculosis for fast molecular identification and genotyping," Brazilian Journal of Microbiology, vol. 42, no. 2, pp. 774-777, 2011.

[28] J. Rudeeaneksin, S. Bunchoo, S. Srisungngam, P. Sawanpanyalert, S. Chamnangrom, A. Kamolwat, P. Thanasripakdeekul, T. Taniguchi, C. Nakajima, and Y. Suzuki, "Rapid identification of Mycobacterium tuberculosis in BACTEC MGIT960 cultures by in-house loopmedicated isothermal amplification," Japanese journal of infectious diseases, vol. 65, no. 4, pp. 306-311, 2012.

[29] Y. Liu, D. Schulze-Makuch, J.-P. De Vera, C. Cockell, T. Leya, M. Baqué, and M. Walther-Antonio, "The Development of an Effective Bacterial Single-Cell Lysis Method Suitable for Whole Genome Amplification in Microfluidic Platforms," Micromachines, vol. 9, no. 8, pp. 367, 2018.

[30] J. Kim, S. H. Jang, G. Jia, J. V. Zoval, N. A. Da Silva, and M. J. Madou, "Cell lysis on a microfluidic CD (compact disc)," Lab on a Chip, vol. 4, no. 5, pp. 516-522, 2004.

[31] M. D. Kulinski, M. Mahalanabis, S. Gillers, J. Y. Zhang, S. Singh, and C. M. Klapperich, "Sample preparation module for bacterial lysis and isolation of DNA from human urine," Biomedical microdevices, vol. 11, no. 3, pp. 671-678, 2009.

[32] M. Islam, A. Shahid, K. Kuryllo, Y. Li, M. J. Deen, and P. R. Selvaganapathy, "Electrophoretic concentration and electrical lysis of bacteria in a microfluidic device using a nanoporous membrane," Micromachines, vol. 8, no. 2, pp. 45, 2017.

[33] F. De Paoli, "Measuring Polydimethylsiloxane (PDMS) Mechanical Properties using flat punch nanoindentation focusing on obtaining full contact," 2015.

[34] D. Li, "Electro-viscous effects on pressure-driven liquid flow in microchannels," Colloids Surfaces A: Physicochemical Engineering Aspects, vol. 195, no. 1-3, pp. 35-57, 2001. 
[35] D. Pfund, D. Rector, A. Shekarriz, A. Popescu, and J. Welty, "Pressure drop measurements in a microchannel," AIChE Journal, vol. 46, no. 8, pp. 1496-1507, 2000.

[36] J. Kim, M. Johnson, P. Hill, and B. K. Gale, "Microfluidic sample preparation: cell lysis and nucleic acid purification," Integrative Biology, vol. 1, no. 10, pp. 574-586, 2009.

[37] J. Kestin, W. A. Wakeham, and C. Y. Ho, Transport properties of fluids: thermal conductivity, viscosity, and diffusion coefficient: Hemisphere Pub, 1988.

[38] F. M. a. C. White, I., "Viscous fluid flow (Vol. 3, pp. 433-434). New York: McGraw-Hill.," 2006.

[39] M. Bahrami, Yovanovich, M.M. and Culham, J.R., "Pressure drop of fully-developed, laminar flow in microchannels of arbitrary crosssection.," 2006.

[40] R. Hu, Li, F., Lv, J., He, Y., Lu, D., Yamada, T. and Ono, N., "Microfluidic analysis of pressure drop and flow behavior in hypertensive micro vessels.," Biomedical microdevices, vol. 17, no. 3, pp. 60, 2015.

[41] M. Akbari, Sinton, D. and Bahrami, M., "Pressure drop in rectangular microchannels as compared with theory based on arbitrary cross section. ," Journal of Fluids Engineering, vol. 131, no. 4, 2009. 